\title{
La tension est-ouest comme ressort dramatique chez deux écrivains francophones d'origine chinoise
}

\section{Florence Gacoin-Marks}

\section{Synopsis}

Dans le présent article, nous nous proposons donc de définir en quels termes la relation « est-ouest » s'articule dans deux romans écrits par des écrivains francophones d'origine chinoise à la fin du XX' $\mathrm{XX}^{\mathrm{e}}$ : Le Jeu de l'eau et du feu de Ya Ding (1990) et Le Dit de Tian-yi de François Cheng (1998). Dans les deux cas, l'expérience de la rencontre entre les cultures française et chinoise est un ressort entre les cultures française et chinoise est un ressort dramatique essentiel et crée dans la littérature créée en France un espace littérature spécifique, une sorte « d'entre-deux » que l'on peut considérer comme un sous-genre littéraire à part entière.

Mots-clés : littératures francophones, imagologie, relations est-ouest, écrivains franco-chinois, Ya Ding, François Cheng, Le Jeu de l'eau et du feu, Le Dit de Tianyi 


\section{INTRODUCTION}

En tant que terre d'accueil choisie par de nombreux exilés, la France a vu sa culture s'enrichir de nouveaux écrivains venus d'ailleurs mais écrivant en français à l'attention du lectorat francophone. Bien qu'étant dans une situation très différente, ces écrivains francophones ont en commun avec les autres, qui vivent hors de France, le fait d'introduire dans la littérature française un ailleurs, celui dont ils viennent, et d'approfondir la réflexion sur les relations entre cet ailleurs et la culture française. ${ }^{1}$

Le cas des écrivains chinois vivant en France et créant en français est particulièrement intéressant du fait que la distance entre les deux cultures impliquées, plus largement entre l'Orient et l'Occident, est suffisamment grande pour permettre une réflexion complète sur tous les aspects constituant une culture au sens large, depuis les plus quotidiens (les us et coutumes) aux plus abstraits (la conception de l'art, la philosophie de la vie). Mieux encore, dans leurs romans, la tension Est-Ouest remplit la fonction de ressort dramatique autour duquel s'organise l'intrigue dans son ensemble.

Dans le présent article, nous nous proposons donc de définir en quels termes la relation « est-ouest » s'articule dans deux romans écrits par des écrivains francophones d'origine chinoise à la fin du XX $\mathrm{XX}^{\mathrm{e}}$ siècle : Le Jeu de l'eau et du feu de Ya Ding (1990) et Le Dit de Tian-yi de François Cheng (1998). Bien qu'elle soit thématisée et interprétée différemment, cette rencontre entre l'Orient et l'Occident au cœur de ces deux œuvres présente un certain nombre de similitudes sur lesquelles il conviendra de s'arrêter.

Mais tout d'abord revenons sur l'intrigue développée dans les deux ouvres étudiées. Le Jeu de l'eau et du feu se présente comme un roman initiatique relatant l'arrivée du héros chinois en France et ses premières années d'exil. Il s'organise autour de trois relations sentimentales, de trois femmes, qui sont autant d'étapes dans son expérience de la vie en Occident. L'expérience s'achève sur une séparation (la troisième femme lui échappe et part pour l'Inde) et une intégration ratée à la culture occidentale. Dans Le Dit de Tian-yi, le narrateur transmet au lecteur le journal d'une de ses connaissances, le peintre Tian-yi, qu'il a connu alors qu'il vivait lui-même « le dur apprentissage de l'exil » en France. Cet exil est relaté dans la partie centrale du livre. Après avoir opéré la synthèse des cultures orientale et occidentale dans ses propres œuvres picturales, le héros rentre en Chine à l'époque de la révolution culturelle.

Dans ces deux romans sur l'exil, la rencontre entre les cultures orientale et occidentale se déroule en trois étapes : la connaissance des stéréotypes chinois sur

1 La présente monographie est le fruit de recherches effectuées dans le cadre du programme de recherche $\mathrm{n}^{\circ} \mathrm{P} 6-0265$, cofinancé à l'aide des fonds publics gérés par l'Agence nationale pour la recherche de la République de Slovénie. 
l'Occident, la rencontre avec la culture occidentale avant le départ pour la France et l'expérience immédiate de la rencontre directe entre l'Orient et l'Occident (expérience du voyage ou de l'exil). Cet axe croissant ou ascendant est également un ressort dramatique important dans les deux œuvres étudiées parlant de l'exil.

\section{CLICHÉS CROISÉS EST-OUEST}

La représentation d'une culture étrangère en littérature est ce que le comparatiste français Daniel-Henri Pageaux appelle une image littéraire, c'est-à-dire « un ensemble d'idées sur l'étranger prises dans un processus de littéralisation, mais aussi de socialisation » (Pageaux 1994, 60). Parmi ces images, il y en a une qui particulièrement forte, c'est celle du stéréotype, appelée aussi cliché.

Le stéréotype tient aussi son importance au fait qu'il s'agit la plupart du temps du premier contact avec l'étranger.

Or, comme l'explique Daniel-Henri Pageaux, le stéréotype repose sur quatre mécanismes :

- sur la transformation des signes culturels en signaux ne renvoyant qu'à une seule interprétation,

- sur la confusion entre l'accessoire (adjectif, attribut) et l'essentiel,

- sur « un abrégé emblématique d'une culture » (généralisation abusive),

- sur la confusion entre Nature et Culture, entre Etre et Faire (Pageaux 1994, 62-63).

On retrouve ces quatre mécanismes dans les stéréotypes présentés dans les romans de François Cheng et Ya Ding.

\section{Stéréotypes des Chinois sur l'Occident (et sur la France en particulier)}

François Cheng et Ya Ding évoquent rapidement les stéréotypes chinois sur la France, mais c'est surtout pour dénoncer la fausse image qu'ils véhiculent.

Ainsi, malgré la Seconde guerre mondiale qui y fait rage, le monde occidental est idéalisé : «Appel de l'Occident. Ou plus exactement de l'Europe. Malgré l'atroce drame qui s'y passait, on ne pouvait s'empêcher de l'idéaliser, d'y voir un sol 'béni des dieux' " (Cheng 1998, 91). De même, Paris est représenté come une " ville lumière », ce qui déçoit le héros à son arrivée dans la capitale française : "Je fus frappé par la couleur terne des rues de Paris, contrastant avec l'image de cette ville lumière que mon imagination avait magnifié » (Cheng 1998, 211). Le charme de Paris est réel, mais il se trouve ailleurs (Cheng 1998, 211). 
Liang, le héros de Ya Ding est nourri de stéréotypes quand il quitte la Chine : il « se souvient du texte que leur professeur leur avait fait apprendre par cœur pendant sa première année de français. 'Le béret sur la tête, la baguette de pain sous le bras, et si possible râleur, telle est l'image du Français moyen'» (Ding 1990, 41). Or, il constate bien vite que « [p] ersonne ne porte de béret sur la tête, ni de baguette de pain sous le bras » (Ding 1990, 41).

L'inadéquation entre les stéréotypes et la réalité a pour effet de déstabiliser le héros qui croyait faire irruption dans un monde connu et se retrouve plongé dans l'inconnu : «-Paris, Paris... Machinalement, Liang se répète ce nom comme celui d'une personne à la fois chère, familière et inconnue »(Ding 1990, 42).

Cette nouvelle image lui donne le vertige, fait d'autant plus ressortir le poids de cet héritage culturel inconnu (Ding 1990, 43). Opaque, la population paraît hostile, perd de son humanité, est décrite comme des « regards froids ", des « visages fermés " et des " pas pressés ». Cette impression d'être face à un " monde d'étrangers » lui fait prendre conscience de son propre statut d'étranger : «Mais non, c'est lui, Liang, l'étranger ". Liang ne sait plus non plus quelle est son identité dans ce monde où il ne sait plus qui sont les êtres humains autour de lui : "Comment est-il perçu par ces étrangers ? Un jeune Japonais ? Un Asiatique ? Ou tout simplement un garçon brun, un rien auquel on ne fait même pas attention ». Et ne sachant plus qui il est, il ne sait plus comment il doit se comporter : «Comment va-t-il réagir face à eux ? Marcher discrètement en rasant les murs ? Garder une allure humble et avenante ? Éviter leur regard ? Ou faut-il se comporter comme il aurait fait dans les rues de Pékin, en chantonnant, les mains dans les poches, en prenant l'air d'être le maître du monde ? » Il ne sait pas comment se positionner par rapport aux autres : « [Q]ue faut-il inspirer à ses interlocuteurs? L'amour, la pitié, ou la force ? Quelle prise avoir sur cette foule inconnue ? » (Ding 1990, 41). Il est « [u] n étranger dans un monde d'étrangers. [Liang] se sent lointain et intrus parmi ces corps humains dont il parle à peine la langue et dont l'esprit secret lui est à jamais fermé »(Ding 1990, 53). Désavoués par la réalité, les stéréotypes des Chinois sur l'Occident sont donc moins une source de connaissance qu'une source de désemparement pour l'étranger en exil.

\section{Stéréotypes des Français sur l'Asie (et sur la Chine en particulier)}

Dans ce cas, il s'agit d'une image littéraire inversée et indirecte, puisque ce sont les écrivains chinois qui la rapportent dans leurs romans. Son importance n'est pas moindre du fait qu'elle renvoie à l'exilé chinois sa propre image déformée par l'incompréhension interculturelle et lui permet de mieux évaluer à la fois ce qui séparent les cultures française et chinoise et quelle est la place qui lui est assignée au départ dans le pays où il est amené à vivre. 
« D'autres fins esprits, se targuant d'être connaisseurs, décrétèrent devant moi ce qu'est la pensée chinoise, la poésie chinoise, l'art chinois. Après les avoir écoutés, j'ai fini par saisir ce qu'ils exigeaient d'un Chinois. Qu'il soit cet être à l'esprit planant, vierge de tourments et dénué d'interrogations, au visage lisse et plat, béatement souriant, fait d'une autre substance que la chair et le sang. Son langage doit être délié, naturel, sans efforts accumulés, sans formes construites, d'une simplicité un peu naïve et son propos doit se ramener à quelque aimable sagesse » (Cheng 1998, 214-215).

Tan-yi déduit de ces stéréotypes - qui ne correspondent pas du tout à sa propre personnalité - le comportement qu'il doit adopter : " je me répétais qu'il faudrait désormais que je m'applique à être chinois, à me conformer à l'idée qu'on se fait d'un Chinois » (Cheng 1998, 215).

Le stéréotype décrit par Ya Ding dans son roman est un peu différent. Un personnage français décrit la Chine en ces termes : «C'est un pays si vaste, si mystérieux, il est rempli de richesse et d'esprit. Les hommes de là-bas sont fins et sensuels ». Confronté à cette généralisation abusive, le héros comprend ce qui sépare l'Est de l'Ouest : " Je ne savais pas comment vous, les Occidentaux, vous nous voyiez; nous sommes si lointains, si différents » (Ding 1990, 152). La généralisation abusive creuse les différences au lieu de les combler.

Fait intéressant : comme le remarque Muriel Détrie, la Chine décrite par les écrivains chinois vivant en France, une Chine rendue atemporelle par l'expérience de l'exil, « correspond plus ou moins à l'image idéale que les Français se font de la Chine traditionnelle sur la foi de certains romanciers français comme José Frèches qui puisent eux aussi leur inspiration dans la Chine historique ou légendaire » $(2004,65)$.

\section{LA RENCONTRE AVEC LA CULTURE OCCIDENTALE : ÉMOI PHYSIQUE, TRANSGRESSION MORALE ET CHOC DES CONTRAIRES}

La Chine pré-communiste de Tian-yi est en contact avec la culture occidentale dont elle apprécie les apports dans tous les domaines. François Cheng revient sur l'histoire de la traduction des littératures occidentales en chinois, rappelant la phrase de l'intellectuel et écrivain Lu Xun conseillant aux jeunes de lire « le moins possible de livres chinois et le plus possible de livres étrangers »(Cheng 1998, 87), précisément pour élargir son horizon.

La véritable première rencontre directe avec la culture occidentale a donc lieu en Chine même, lorsque les jeunes Chinois se familiarisent avec les produits et les œuvres de cette culture, depuis le fumet des viennoiseries jusqu'aux romans 
de Balzac, en passant par les tableaux de Cézanne ou Van Gogh et les symphonies de Dvořák ou Beethoven. Cette culture se présente tout d'abord comme une culture touchant tous les sens, qui bouleverse le corps, comme une culture transgressive, voire interdite et enfin comme une culture bouleversant l'esprit par sa représentation de la réalité, très contrastée par rapport à celle proposée par la culture chinoise.

\section{Une culture très "physique ", mais transgressive, voire interdite}

Tian-yi, le héros de François Cheng, découvre la culture occidentale dès son enfance. Celle-ci se présente à lui comme une culture très " physique ", s'adressant à tous ses sens. Ainsi, il découvre les tableaux du Louvre grâce à des cartes postales rapportées par une tante :

Parmi les choses qu'elle avait rapportées de France et qu'elle nous montra en vrac, mon regard s'arrêta sur des cartes reproduisant des œuvres du musée du Louvre $[\ldots]$, peintures représentant des nus, notamment deux tableaux, ayant pour figure principale une femme nue vue de dos. [...] Oui, ces femmes nues, au cœur superbe, les premières que je voyais de ma vie, si étrangères et qui pourtant avaient instantanément remué en moi le sang le plus intime, comment les oublierais-je désormais ? (Cheng 1998, 47-48).

Physique, la culture occidentale est également considérée comme licencieuse et transgressive dans ce monde évitant toute mise à nu du corps comme des sentiments. Ainsi, les cartes postales du Louvre sont « ramassées en hâte par les grandes personnes présentes, surprises, outrées » (Cheng 1998, 47), comme si ces tableaux étaient des images pornographiques. Et il ne fait aucun doute qu'une partie de leur charme vient de cette transgressivité. Dans tous les cas, elles restent gravées à jamais dans l'esprit du personnage, elles l'ont ému « au point de tracer des sillons dans mon imaginaire "(Cheng 1998, 48). Fait amusant : il est plus impressionné par les dos nus plus que par les seins, partie du corps qu'il a déjà vue à plusieurs reprises quand les femmes chinoises allaitaient leur enfant.

On retrouve cette idée de la transgressivité de la culture occidentale plus tard, au cinéma :

Les scènes d'amour, avec des femmes montrant sans retenue leurs attraits, et les baisers prolongés transperçaient la carapace de pudeur des Chinois jusqu'à la douleur. Dans le noir, les spectateurs subissaient le choc d'abord avec gêne et stupeur, puis avec ravissement : ils sentaient le sang bouillir dans leurs veines et les fanges enfouies de leur imaginaire remonter à la surface (Cheng 1998, 116). 
Là encore, l'image passe par le corps pour frapper l'imaginaire.

Plus prosaïquement, la culture étrangère parle tant à l'odorat qu'à la vue du jeune garçon quand il passe à côté d'une boulangerie française : «Moment de pur ravissement pour moi ! Enveloppé de la chaude odeur retrouvée, je nétais plus qu'yeux face aux choses lumineuses exposées dans les vitrines » (Cheng 1998, 94). Cette impression est complétée par un autre sens, le goût, subjugué par une " saveur exotique » indéfinissable, mais « conforme à ce que j'avais intensément imaginé. » Il tire de cette première expérience un principe de vie qui ne le quittera plus : "En somme, la satisfaction de tout désir est dans le désir lui-même » (Cheng 1998, 96). Il retrouvera plus tard cette idée chez Gide, dont la lecture permet aux jeunes Chinois d'éprouver dans leur corps « l'expression favorite de l'écrivain 'la soif étanchée' ", l'idée que "l'accomplissement de l'homme est dans le désir luimême » (Cheng 1998, 132). Par ailleurs, il est désormais « préparé à accueillir tout ce qui venait de plus loin» (Cheng 1998, 96).

Plus tard, l'intrusion de la culture occidentale en plein cœur de la Chine pré-communiste touche un nouveau sens du jeune homme : l'ouïe. En découvrant La Symphonie pastorale, Tian-yi y perçoit des « accents si souverains et conquérants ". Tandis que la musique chinoise est " retenue et confidentielle, souvent plaintive ", la musique occidentale « n'accompagne pas la nature; il en déchire la peau, en transperce la chair pour ne devenir la pulsation même ». C'est donc dans son corps que le jeune homme ressent la culture occidentale : "Cette fois-ci, j’étais atteint au plus intime, au creux de mes entrailles » (Cheng 1998, 98)

La culture occidentale se définit donc avant tout par sa surabondance physique : elle est « matière » et " glorifie le visible », se différenciant ainsi de la culture extrême-orientale qui " par réductions successives, cherche à atteindre l'essence insipide où l'intime de soi rejoint l'intime de l'univers »(Cheng 1998, 96). Cette très grande différence a en partie pour origine le fonctionnement différent des langues chinoise et européennes. Comme l'explique François Cheng dans l'un de ses essais, "si les idéogrammes chinois suggèrent les choses qu'ils désignent par leurs graphies, les mots français, phonétiques, suggèrent les choses désignées par leur son. [...] Chaque mot possède un rythme, une mélodie, capable de susciter en moi une image souvent très incarnée » (Cheng 2015, 29-30).

Après l'avènement du maoïsme intervient la première censure de la culture occidentale : «Pour ce qui concerne l'art occidental, on n'étudie plus que les peintres dont les thèmes paraissent 'sûrs', qui ont un contenu social : Le Nain, Millet, Delactroix pour avoir traité d'un rare sujet révolutionnaire, Courbet pour avoir participé à la Commune de Paris » (Cheng 1998, 314). La sélection se fait selon des critères purement politiques, et non esthétiques, ce qui met fin au pouvoir de l'influence occidentale sur la culture chinoise. 


\section{Une culture qui bouscule la représentation de la réalité humaine}

Sur un plan plus spirituel, la culture occidentale bouscule la représentation de la réalité humaine à laquelle les Chinois ont été habitués par leur propre culture.

Dans le roman de François Cheng, le jeune peintre Tian-yi a le sentiment que la culture chinoise de son temps connaît une période de stagnation, quelle n'apporte plus rien de nouveau. Selon lui, « [p] our le moment, le salut vient d'ailleurs, de l'étranger. Et en premier lieu, de l'Occident. C'est là quont été formulées les interrogations et accomplies des créations que nous n'avons pas faites et que nous ne pouvons pas contourner » (Cheng 1998,103). Cependant, le héros avertit le lecteur qu'il ne s'agit pas de prêter allégeance aveuglément à un Occident dont les travers ont apporté tant de souffrances dans le monde, y compris en Asie, mais de se plonger dans les œuvres des «vrais créateurs, de ceux qui tentent justement de dévoiler le vrai. Leurs cris ou leurs chants, dans la liberté conquise, proprement inouïs pour nous, déchirent notre horizon. Oui, il faut bien cet extrême autre pour nous secouer, pour nous arracher à la partie dégénérée, pourrie, de nos racines " (Cheng 1998, 104).

Comme en Sibérie orientale, dans le roman Au temps du fleuve Amour (1994) de l'écrivain francophone d'origine russe Andreï Makine, c'est au cinéma que la culture occidentale apparaît dans toute sa différence, dans toute sa vitalité (Cheng 1998, 115). Le film américain des années trente, dans toute son exubérance, fascine les jeunes chinois. La culture chinoise semble statique, c'est donc la vitalité et le dynamisme de la culture occidentale au cours de l'histoire (Ding 1990, 74).

Changement constant, profusion des idées, c'est ce qui séduit le jeune Chinois, notamment chez Van Gogh : « je me sentais proche de Van Gogh, de cette représentation fragmentée des formes, de cette alchimie audacieuse des couleurs et de cette vision personnelle captée au cœur même du temps vécu ! Son œuvre résonnait en moi comme un appel fraternel. Ce bas monde, aussi provisoire soit-il, demande à être exprimé. Malgré mon dénuement, je le ferai par la peinture » (Cheng 1998, 105). Et c'est d'ailleurs ce qui va le pousser à intégrer cette culture par le biais de sa propre activité créatrice : «Avec toute la sympathie que je nourrissais pour Cézanne, pour Kandinsky, pour Klee, je devrais pouvoir sans trop de mal me glisser parmi mes contemporains, dans cet Occident en proie aux changements rapides, qui ne jurait que par la nouveauté » (Cheng 2015, 263).

Notons que, au départ, le jeune Tian-yi s'initie à la culture occidentale par l'intermédiaire de la culture la moins éloignée géographiquement : «La musique de Dvorak, originaire d'Europe centrale, avait pour effet de rendre moins lointain d'Occident extrême, en sorte que la terre russe paraissait presque proche » (Cheng 1998, 102).

Comme nous l'avons déjà vu, la littérature française, de Gide à Rimbaud, montre à Tian-yi que « l'accomplissement du désir de l'homme se trouve dans le 
désir lui-même » (Cheng 1998,132). Cette vision des choses va par la suite conditionner sa lecture critique de Proust : "Contrairement à Proust, j’aurais écrit : 'À la recherche du temps à venir '. La loi du temps, du moins ma loi à moi [...] n'était pas dans l'accompli, dans l'achevé, mais dans le différé, l'inachevé » (Cheng 1998, 206). Cet enseignement livresque permet au héros de mieux comprendre et exprimer l'histoire vécue avec l'Amante, la femme qu'il désirera sans jamais la posséder, rappelle un thème récurrent chez Gide, celui de l'amour inassouvi, au centre de $L a$ Porte étroite, notamment.

La culture occidentale exerce donc une influence d'autant plus considérable qu'elle fait bouger les lignes, apporte une réponse aux interrogations des jeunes Chinois sur la vitalité et le désir propres à leur âge et dont la culture chinoise, sclérosée par les vicissitudes de l'histoire, ne parle pas. Cette culture vient donc combler un manque.

\section{Confrontation des mentalités: différences concernant les relations sociales}

Les deux romans étudiés abordent le thème de l'exil. Munis des connaissances sur la culture occidentale acquises avant le départ de Chine, le personnage nouvellement arrivé à Paris se trouve confronté aux différences culturelles. Dans le roman de Ya Ding, cette question occupe une place centrale, tandis qu'elle n'est pratiquement pas abordée par François Cheng dans Le Dit de Tian-yi.

Le récit du séjour de Liang à Paris est émaillé de constatation concernant les différences entre l'Orient et l'Occident en matière de relations sociales. Liang finit par en déduire qu' «il a bien des choses à apprendre et à avaler s'il veut vivre en France!»(Ding 1990, 278). Les conventions sociales diffèrent beaucoup. En voici un exemple parmi tant d'autres :

[E]n Occident, même entre amis, on ne se raconte pas tout, lui avait dit son professeur de français dès sa première année. C'est juste, d'ailleurs ; en Chine, tout est si public que chacun est pour l'autre comme une ampoule de mille kilowatts, et ne laisse aucune ombre sur sa vie privée (Ding 1990, 201).

Parfois, ces différences comportementales fascinent Liang par leur caractère sophistiqué : «Les Français ont l'habitude d'être en retard, pour se faire désirer, pour se montrer désintéressés, pour faire avancer les autres vers eux... Quel art des relations, quelle subtilité d'esprit ! (Ding 1990, 277).

Mais, en d'autres occasions, la vision occidentale des choses déçoit le jeune chinois. Constatent que personne ne songe à le retenir de faire une bêtise, il dénonce l'hypocrisie consistant à faire mine de respecter la liberté de l'autre : « Alors 
c'est ça, l'Occident. On lâche facilement un ami sur la mauvaise voie, avec la meilleure des bonnes consciences, puisqu'on respecte l'indépendance de l'autre » (Ding 1990, 269).

Dans tous les cas, ces différences entravent l'intercompréhension entre Liang et les Français qu'il est amené à côtoyer à Paris : "Il n'est vraiment pas dans son pays. Au moment où il croit avoir compris quelque chose, tout change " (Ding 1990, 201). Le jeune chinois ne sait comment interpréter l'attitude de certains de ses interlocuteurs. Par exemple, une femme française qui appelle régulièrement un garçon est-elle amoureuse (comme le serait une femme chinoise se conduisant de la même manière) ? De même, un jeune homme peut-il téléphoner souvent à une fille sans avoir l'air de l'importuner (ce qui est « très mal vu en Chine) ?

Les relations sociales sont également compliquées par les différences linguistiques. Ainsi, pour s'excuser de ne pas avoir assez pris en considération un éditeur parisien, le personnage se souvient de ce qu'il dirait en chinois, mais reste muet en français : "Mes yeux aveugles ne savent pas reconnaître la montagne Tai, disent toujours les Chinois pour s'excuser devant les grands personnages qu'ils ont négligés par ignorance. Mais comment traduire cela en français ? " (Ding 1990, 252253). De même, face à l'utilisation généralisée du pronom possessif en français, le personnage finit par comprendre que cette catégorie de mots « n'a apparemment pas le même sens qu'en chinois » (Ding 1990, 267).

En réalité, la différence la principale différence entre les mentalités occidentale et chinoise semble d'ordre philosophique : le caractère entier des Occidentaux, où l'opposition entre le « oui » et le « non » est très nette, s'oppose catégoriquement à la recherche incessante de l'équilibre, du compromis, propre à la culture chinoise. Le personnage prend conscience de cette différence fondamentale en observant la forme du loquet d'une porte :

[I]1 a été trompé par la forme du loquet qui est d'une courbe verticale. C'est un signe qui réveille chez les Chinois leur instinct de prendre la contrepartie, et d'appuyer vers le bas pour rééquilibrer les choses. [...] Cela veut dire que l'instinct des Français est de continuer ce qui a déjà ét[é] fait dans un premier mouvement. Ils ont la mentalité de pousser les choses vers l'extrême, tandis que chez nous, se dit Liang, on ramène plutôt les choses vers leur état primitif ; deux esprits différents, l'un allant vers l'extrême, et l'autre vers l'équilibre (Ding 1990, 53).

Plus tard, il en fait la triste expérience lorsqu'il vexe un éditeur parisien par son indécision, interprétée comme du désintérêt, et non comme une marque de sérieux et de modestie comme en Chine (Ding 1990, 253). La rencontre directe avec la culture occidentale est source de difficultés dans tous les domaines de l'existence. 


\section{DÉNOUEMENT DE LA RENCONTRE EST-OUEST : RECONCILIATION DES CONTRAIRES OU ILLUSIONS PERDUES ?}

La rencontre est-ouest, ressort dramatique essentiel des deux œuvres romanesques étudiées, connaît deux dénouements très distincts.

\section{L'échec de l'intégration: l'impossible cohabitation des contraire (Liang)}

Les difficultés que connaît Liang, le personnage du Jeu de l'eau et du feu, sont telles que le jeune homme « ouvre sa bouche, il a envie de cracher, cracher hors de lui son sang ou son âme chinoise ! (Ding 1990, 211).

Dans son cas, l'issu du processus est malheureuse. Le seul moment de bonheur total connu par le personnage se produit lors d'un séjour au ski, dans le téléphérique : "Dans cette ascension à ciel ouvert, Liang plonge dans un autre monde ; quittant la France, quittant cette terre, il retrouve la sienne, identique à lui, à son âme » (Ding 1990, 236). Un peu comme le héros d'Illusions perdues de Balzac, le héros n'a rien fait comme il le fallait pour réussir sur le plan professionnel et sentimental et se retrouve à la fin du roman dans le plus grand dénuement. Ayant échoué en tant quécrivain, il perd également la femme avec laquelle il pensait effectuer la synthèse entre l'Orient et l'Occident. Trop transformé par son expérience pour pouvoir réintégrer la société chinoise et trop peu transformé pour avoir réussi à s'intégrer à la société française, il n'a plus d'identité : « Il n'existe plus, ni pour la Chine, ni pour la France !» (Ding 1990, 289), « [il] n'a plus de chez lui, il n'a plus de patrie » (Ding 1990, 339). Dans son désespoir, le personnage imagine les conséquences de son acculturation sur son père, le voyant en pensée « [r]avagé par les difficultés de la vie, confit dans sa faiblesse, il se reconnaît plus ce fils occidentalisé, traître à la sagesse, à la tradition millénaire, habitué au confort, au goût étranger, et aspirant à la passion. Un fils perdu !» (Ding 1990, 339). La chaîne de la tradition - si importante dans la culture chinoise - se trouve brisée.

\section{La réconciliation des cultures chinoise et occidentale grâce à l'art (Tian-yi)}

Au départ, Tian-yi, le héros de François Cheng, connaît des difficultés analogues à celles rencontrées par Liang. Perdu dans Paris, il ne reprend espoir qu'après avoir retrouvé en plein cœur de la capitale française une nourriture familière (une soupe chinoise aux nouilles). Il s'écrit alors : «Oui, j’apprendrai à 
aimer cette ville où je vais vivre un certain temps. J'apprendrai à aimer ce pays qui se trouve au cœur de l'Europe occidentale. Ce sera une longue initiation. En attendant, il faut passer - je le pressens, je le sais déjà - par le purgatoire, sinon par l'enfer» (Cheng 2015, 212).

C'est le cheminement artistique qui sauvera le jeune peintre du sort malheureux subi par Liang. Accaparé par l'idée de réconcilier tradition chinoise et peinture occidentale, il ne s'arrêtera pas aux difficultés liées à l'exil mais fera sur lui-même un travail de fond pour effectuer la synthèse entre les deux cultures en présence. Tout part des paroles du maître chinois qui enseigne ce qu'il maîtrise, la tradition picturale chinoise : « D'abord, donc, posséder ce que la tradition offre de meilleur" (Cheng 2015,231). La connaissance de sa propre culture n'est pas " un carcan ", " un enfermement sur soi ", elle est la base sur laquelle fonder sa liberté et se préparer « à la vraie rencontre avec un autre, à l'affronter sans se perdre » (Cheng 2015, 176). Le jeune chinois ne s'éloignera pas de ce chemin : "M'étant déjà familiarisé avec la méthode chinoise des 'trois couches et cinq points', j'étudiais avec application aux Beaux-Arts la manière occidentale de dessiner les portraits. » (Cheng 2015, 231). Il en est de même quand il admire la peinture italienne lors d'un court séjour à Florence et à Rome : « C'était fort de ma propre tradition et de mon expérience à Dunhuang que je parvins finalement à affronter cette autre peinture ; faute de quoi je me serais senti écraser » (Cheng 2015, 243). C'est la culture étrangère qui permet au personnage de renouer avec ce qui fait l'essence de sa propre culture et d'apprécier à sa juste valeur - après l'avoir minoré - le rôle spécifique de cette dernière dans l'histoire (Cheng 2015, 250). C'est parce qu'il a réussi dans son esprit et ses propres œuvres la synthèse entre les cultures chinoise et occidentale que Tian-yi sort triomphant de son expérience d'exil interrompu par un retour en Chine pour raisons sentimentales.

Néanmoins, on peut se demander si cette expérience réussie ne causera pas indirectement sa perte en l'empêchant de s'adapter aux restrictions et à l'enfermement caractérisant l'ère de la Révolution culturelle. Comme l'écrit Muriel Détrie, « [n]ourris de références françaises ou marqués par leur séjour en France, les personnages revendiquent le droit à la liberté, à l'individualisme ou à l'amour, se coupant par là de leur pays d'origine où ces mêmes droits ne sont guère reconnus » (2004, 65). Lors du retour en Chine, Tian-yi doit faire face à cette saisissante régression.

\section{CONCLUSION}

Les Chinois de l'ère contemporaine ont en commun d'être en contact indirect ou direct avec la culture occidentale. Comme le rappelle Tian-yi : « [j]'étais ce 
Chinois du $\mathrm{XX}^{\mathrm{e}}$ siècle, depuis toujours ballotté, provoqué par la Chine, provoqué par l'Occident, provoqué par la vie » (Cheng 2015, 262-263). Bien que vécue différemment, l'expérience de la rencontre entre les cultures chinoise et française est un ressort dramatique important dans les romans écrits par les écrivains francophones d'origine chinoise. Comme le note Muriel Détrie, « l'écartèlement entre deux cultures, deux pays, deux systèmes de valeurs, est un thème récurrent » (2004, 65). Ces romans ont en commun avec les autres romans francophones le fait d'introduire un ailleurs dans la vie littéraire française et de créer, comme le montre Lena Besinger dans son ouvrage, un espace romanesque spécifique, un entre-deux, et donc un genre littéraire "nouveau ", émergeant. Cependant, à l’ère de la mondialisation, les différences culturelles s'estompent et les contacts interculturels se multiplient. Nous pouvons donc nous demander si les œuvres étudiées ne sont pas, en quelque sorte, les derniers vestiges d'un monde en voie de disparition. Bien qu'on puisse se réjouir que la compréhension entre les peuples soit facilitée, on ne peut que déplorer l'appauvrissement et l'uniformisation culturels induits par cette nouvelle situation internationale.

\section{BIBLIOGRAPHIE}

\section{Bibliographie primaire}

Cheng, François : Le Dit de Tian-Yi, Paris, Le Livre de Poche, 1998.

Ding, Ya : Le Jeu de l'eau et du feu, Paris, Flammarion/Stock, 1990.

\section{Bibliographie secondaire}

Bisinger, Lena : Rencontre interculturelle dans le roman franco-chinois. Invitation au voyage d'un genre émergent, Berlin, Logos, 2016.

Cheng, François : Le Dialogue, Desclée de Brouwer, 2002.

Cheng, François : Entretiens avec Françoise Siri, Paris, Albin Michel, 2015.

Delbart, Anne-Rosine : Les Exilés du langage. Un siècle d'écrivains français venus d'ailleurs (1919-2000), Limoges, Pulim, 2005.

Detrie Muriel : «Existe-t-il un roman chinois francophone ? ", Le Magazine Littéraire, n 429,2004 , p. 65-66.

Pageaux, Daniel-Henri : La Littérature générale et comparée, Paris, Armand Colin, 1994.

Silvester, Rosalind / Thouroude, Guillaume (dir.) : Traits chinois / lignes francophones. Ecritures, Images, Cultures, Les Presses de l'Université de Montréal, coll. «Sociétés et Cultures d'Asie», 2012. 
Xiang, Weiwei : Interculturalité et intermédialité chez les auteurs francophones chinois. Thèse de doctorat, Université de Strasbourg, 2017.

Zhang, Yinde : "François Cheng ou dire la Chine en français ", Revue de Littérature comparée, 2007/2, pp. 141-152 (dans l'article : Zhang, 2007a).

Florence Gacoin-Marks

Faculté des lettres de l'Université de Ljubljana florence.gacoin-marks@guest.arnes.si

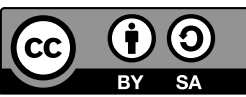

\section{Napetost Vzhod-Zahod kot dramski vzgib za dva frankofonska pi- satelja kitajskega izvora}

V pričujočem članku razpravljam o značilnostih odnosom med Vzhodom in Zahodom $\mathrm{v}$ dveh romanih francoskih pisateljev kitajskega rodu, ki sta nastala ob koncu 20. stoletja: Le Jeu de l'eau et du feu [Igra vode in ognja] Ye Dinga (1990) in Le Dit de Tian-yi [Tianyin izrek] Françoisa Chenga (1998). V obeh primerih je soočenje francoske in kitajske kulture ključna dramska sprožilec. Poleg tega ustvarja v francoski književnosti, ki je nastala v Franciji, specifičen literarni prostor, ki ga lahko imamo celo za samostojno romaneskno podvrsto.

Ključne besede: frankofonske književnosti, imagologija, zahodno-vzhodni odnosi, francosko-kitajski pisatelji, Ya Ding, François Cheng, Le Jeu de l'eau et du feu, Le Dit de Tian-yi 